\title{
INVESTIGATION ON ADAPTATION USING DIFFERENT DISCRIMINATIVE TRAINING CRITERIA BASED LINEAR REGRESSION AND MAP
}

\author{
Bo Zhu, Zhi-Jie Yan, Yu Hu, Zhi-Guo Wang, Li-Rong Dai, Ren-Hua Wang \\ iFlytek Speech Lab, University of Science and Technology of China, Hefei \\ bozhu@mail.ustc.edu.cn yanzhijie@ustc.edu \{yuhu,zgwang $\} @$ iflytek.com $\{$ lrdai,rhw $\} @$ ustc.edu.cn
}

\begin{abstract}
This paper presents a comparison and evaluation between the conventional maximum likelihood estimation based adaptation and different discriminative adaptation criteria. The performance of different LR and MAP adaptation are compared respectively, and the strategies of first applying LR then MAP based on both MLE and DT criteria are evaluated. The effect of the amount of available data for adaptation is also compared in our experiments. The experiment results of 863 and Tsinghua mandarin evaluation tasks suggests that the process of first applying MWCE-LR then MWCE-MAP can achieve the best performance.
\end{abstract}

Index Terms - Adaptation, Discriminative training, Minimum word classification error, Speech recognition

\section{INTRODUCTION}

Adaptation for the target environment or speaker is a very important area in the state-of-the-art automatic speech recognition (ASR) systems, when there is fairly limited amount of adaptation data can be used. Maximum Likelihood Linear Regression (MLLR)[1] has been widely used for model-space adaptation in both supervised and unsupervised modes, the Maximum a-posteriori (MAP)[2] has also been widely applied in many tasks. It has been proved that both of them are able to give good performance.

Discriminative Training (DT) criteria, such as MCE, MMI, MPE, are able to produce solid and consistent performance improvement in both small and large vocabulary speech recognition tasks. In [3], we introduced a novel word-level DT criterion, namely Minimum Word Classification Error (MWCE) which localizes conventional string-level MCE loss function to word level, then a more direct measure of empirical word classification error can be approximated and minimized. In recent years, the discriminative training criteria have been successfully applied to estimate the transform matrices [4][5][6], and they have also been combined a prior distribution with the statistics required for MMI(MPE) estimation while applying adaptation, which are denoted as MMI(MPE)-MAP[7].

In this paper, we introduce a unified Linear Regression (LR) and MAP adaptation framework based on MWCE and the other DT criteria. Because all these criteria we realized share most of the implementation details in a common framework, it can provide a fair evaluation of these different criteria. In our experiments, these aspects are evaluated and compared: 1) The performance of different MLE based and DT based adaptation criteria on Mandarin recognition tasks; 2) The affect of the amount of available adaptation data; 3) Two different evaluation sets of 863 and TsingHua Mandarin are selected to validate these different adaptation criteria and the combination modes of them.
The rest of this paper is organized as follow: In Section 2, the proposed MWCE criterion is briefly introduced. In Section 3, the unified LR and MAP adaptation framework based on MWCE criterion is introduced. In Section 4, the experimental results of evaluation and comparison of these different adaptation criteria are presented. Finally in Section 5, we will draw our conclusions.

\section{MINIMUM WORD CLASSIFICATION ERROR CRITERION}

There is a mismatch between the string-level MCE[8] and word-level performance evaluation measurement. Minimizing string error can only lead to a minimization of word error in a quite indirect way. A new criterion which focuses on word-level error can provide a more effective way to optimize our ultimate goal of WER.

Suppose the reference word sequence of the $r_{t h}$ training utterance is consisted of $N_{r}$ words, i.e., $W_{r}=\left\{w_{r}^{1}, w_{r}^{2}, \ldots, w_{r}^{N_{r}}\right\}$. For each reference word $w_{r}^{n}$, we could define the correct string set $\mathcal{M}_{w_{r}^{n}}^{\mathcal{K}}$ and incorrect string set $\mathcal{M}_{w_{r}^{n}}^{\mathcal{J}}$ such that:

$$
\begin{array}{r}
\forall W \in \mathcal{M}_{w_{r}^{n}}^{\mathcal{K}}, \exists w \in W, w \equiv w_{r}^{n}, \\
\forall W^{\prime} \in \mathcal{M}_{w_{r}^{n}}^{\mathcal{J}}, \forall w^{\prime} \in W^{\prime}, w^{\prime} \neq w_{r}^{n} .
\end{array}
$$

In Eq. (1), $w \equiv w_{r}^{n}$ means that we restrict the word $w$ to have a same label and same time alignment as the reference word $w_{r}^{n}$. Therefore, the correct string set $\mathcal{M}_{w_{r}^{n}}^{\mathcal{K}}$ will include all the dynamic programming decoding strings that pass through a "matched" word $w$ for the corresponding interval of speech. Conversely, the incorrect string set $\mathcal{M}_{w_{r}^{n}}^{\mathcal{J}}$ is consisted of all strings that do not pass any "matched" word for $w_{r}^{n}$. Obviously, $\mathcal{M}_{w_{r}^{n}}^{\mathcal{K}} \cap \mathcal{M}_{w_{r}^{n}}^{\mathcal{J}}=\emptyset$, and $\mathcal{M}_{w_{r}^{n}}^{\mathcal{K}} \cup \mathcal{M}_{w_{r}^{n}}^{\mathcal{J}}=\mathcal{M}$. So the discriminant functions for each string set can be formulated as:

$$
g_{\mathcal{K}}(\theta)=\log \left[\frac{1}{\left|\mathcal{M}_{w_{r}^{n}}^{\mathcal{K}}\right|} \sum_{W \in \mathcal{M}_{w_{r}^{n}}^{\mathcal{K}}} p_{\theta}^{\alpha}\left(X_{r} \mid W\right) \cdot p^{\alpha}(W)\right]^{1 / \alpha},
$$

and

$$
g_{\mathcal{J}}(\theta)=\log \left[\frac{1}{\left|\mathcal{M}_{w_{r}^{n}}^{\mathcal{J}}\right|} \sum_{W^{\prime} \in \mathcal{M}_{w_{r}^{n}}^{\mathcal{J}}} p_{\theta}^{\alpha}\left(X_{r} \mid W^{\prime}\right) \cdot p^{\alpha}\left(W^{\prime}\right)\right]^{1 / \alpha} .
$$

The misclassification measurement and the loss function can be embedded into the unified DT criterion which can be rearranged as the sum of smoothed word errors on the training data:

$\mathcal{F}_{\text {MWCE }}$

$$
=\sum_{r=1}^{R} \sum_{n=1}^{N_{r}} f\left[\log \frac{\sum_{W \in \mathcal{M}_{w_{r}^{n}}^{\mathcal{K}}} p_{\theta}^{\alpha}\left(X_{r} \mid W\right) \cdot p^{\alpha}(W)}{\sum_{W^{\prime} \in \mathcal{M}_{w_{r}^{n}}^{\mathcal{J}}} p_{\theta}^{\alpha}\left(X_{r} \mid W^{\prime}\right) \cdot p^{\alpha}\left(W^{\prime}\right)}\right],
$$


where $f(z)=\frac{-1}{\left(1+e^{2 \rho z}\right)}$ keeps unchanged as string-level MCE defined in the unified framework[9] to maximize the unified criterion for parameters optimization of this negative loss function.

\section{ADAPTATION BASED ON MWCE}

The process of solving the matrices for LR transform and the parameters updated with MAP based on MWCE criterion is presented in the following sections. Although the objective function of MWCE criterion in Eq. (4) is a little different from the unified one defined in [9], for the items related to the sum of the words amount $n$ in each sentence, this solving process can be easily extended for the other DT criteria.

\subsection{MWCE-based discriminative linear transformation}

As the same definition of standard MLLR, MWCELR is also applied to adapt the $m^{t h}$ Gaussian mixture component's extended mean vector with matrix A and bias $b, \hat{\mu}_{m}=W \xi_{m}$, where $\xi_{m}=\left[1 \mu_{m}^{T}\right]^{T}$, $W=[b A]$, and the diagonal variance transform $H$ can be estimated upon the updated mean vector $\hat{\mu}_{m}$. The estimation of diagonal variance transform can be modified as $\hat{\sum}_{m}=H^{T} \sum_{m} H$, $\hat{\sum}_{m}^{-1}=L_{m}^{T} H^{-1} L_{m}$, in which $L_{m}$ is the Choleski factor of $\sum_{m}^{-1}$. We aim to reach a good estimation of $W$ and $H$ with MWCE criterion.

Since it is difficult to optimize the derivation of $\mathcal{F}_{\text {MWCE }}$ directly, we select a simply defined auxiliary function to optimize the objection function instead: $\left.\frac{\partial}{\partial \hat{\lambda}} \mathcal{G}(\hat{\lambda}, \lambda)\right|_{\hat{\lambda}=\lambda}=\left.\frac{\partial}{\partial \hat{\lambda}} \mathcal{F}(\hat{\lambda})\right|_{\hat{\lambda}=\lambda}$, where $\lambda$ refers to the original parameters and $\hat{\lambda}$ represents the newly estimated ones. As the auxiliary function is weak-sense[10], a smoothing function associates with the initial adapted model parameters is added to improve the stability of training to overcome the negative terms in the objective functions, then the auxiliary function of the transform matrix estimation for the parameters in the same regression class can be defined as:

$$
\begin{aligned}
& \mathcal{G}_{\mathrm{MWCE}}(\lambda, \hat{\lambda}) \\
= & \sum_{m} \sum_{r=1}^{R} \sum_{n=1}^{N_{r}} \sum_{t=t_{n}^{s}}^{t=t_{n}^{e}} \gamma_{m}^{\mathrm{num}}(t) f^{\prime}\left(\gamma_{m}\right) \log \mathcal{N}\left(o_{t} \mid \hat{W} \xi_{m}, \hat{H}^{T} \Sigma_{m} \hat{H}\right) \\
- & \sum_{m} \sum_{r=1}^{R} \sum_{n=1}^{N_{r}} \sum_{t=t_{n}^{s}}^{t=t_{n}^{e}} \gamma_{m}^{\mathrm{den}}(t) f^{\prime}\left(\gamma_{m}\right) \log \mathcal{N}\left(o_{t} \mid \hat{W} \xi_{m}, \hat{H}^{T} \Sigma_{m} \hat{H}\right) \\
& +\sum_{m} D_{m}\left[-\frac{1}{2}\left(\log \left|\hat{\Sigma}_{m}\right|\right.\right. \\
& \left.\left.+\left(W \xi_{m}-\hat{W} \xi_{m}\right)^{T} \Sigma_{m}^{-1}\left(W \xi_{m}-\hat{W} \xi_{m}\right)+\sum_{m} \hat{\Sigma}_{m}^{-1}\right)\right] .
\end{aligned}
$$

The last item in Eq. (5) is the smoothing function to improve the stability of training. While setting the derivation of the auxiliary function to zero, $\frac{\partial}{\partial \hat{W}} \mathcal{G}_{\mathrm{MWCE}}(\lambda, \hat{\lambda})=0$, it can be solved in the same way as standard MLLR to find out the solution of the updated transform matrix $\hat{W}$, and the initial matrix $W$ can be set to be an identity matrix.

The $i^{t h}$ row's solution of the linear transform matrix $\hat{W}$ is:

$$
\begin{gathered}
\hat{w}_{i}=z_{i} G_{i}^{-T} \\
z_{i}=\sum_{m} \frac{1}{\sigma_{m i}^{2}}\left(\theta_{m}^{\text {num }}\left(\mathcal{O}_{i}\right)-\theta_{m}^{\text {den }}\left(\mathcal{O}_{i}\right)+D_{m} \tilde{\mu}_{m i}\right) \xi_{m}^{T},
\end{gathered}
$$

$$
G_{i}=\sum_{m} \frac{1}{\sigma_{m i}^{2}}\left(\gamma_{m}^{\text {num }}-\gamma_{m}^{\mathrm{den}}+D_{m}\right) \xi_{m} \xi_{m}^{T} .
$$

In Eq. (6), $\tilde{\mu}_{m}$ is the adapted mean vector with the initial transform $W$, and the other statistics have the following forms:

$$
\begin{aligned}
\gamma_{m}^{\text {num }} & =\sum_{r=1}^{R} \sum_{n=1}^{N_{r}} \sum_{t=t_{n}^{s}}^{t=t_{n}^{e}} \gamma_{m}^{\text {num }}(t) f^{\prime}\left(\gamma_{m}\right), \\
\theta_{m}^{\text {num }}(\mathcal{O}) & =\sum_{r=1}^{R} \sum_{n=1}^{N_{r}} \sum_{t=t_{n}^{s}}^{t=t_{n}^{e}} \gamma_{m}^{\text {num }}(t) f^{\prime}\left(\gamma_{m}\right) o(t) ; \\
\gamma_{m}^{\text {den }} & =\sum_{r=1}^{R} \sum_{n=1}^{N_{r}} \sum_{t=t_{n}^{s}}^{t=t_{n}^{e}} \gamma_{m}^{\text {den }}(t) f^{\prime}\left(\gamma_{m}\right), \\
\theta_{m}^{\text {den }}(\mathcal{O}) & =\sum_{r=1}^{R} \sum_{n=1}^{N_{r}} \sum_{t=t_{n}^{s}}^{t=t_{n}^{e}} \gamma_{m}^{\text {den }}(t) f^{\prime}\left(\gamma_{m}\right) o(t) .
\end{aligned}
$$

In Eq. (7) and (8), $t=t_{n}^{s}$ and $t=t_{n}^{e}$ denote the start time and end time of the word $w_{r}^{n}$ respectively, $f^{\prime}\left(\gamma_{m}\right)$ is the derivation of $f$ in Eq. (4), $\gamma_{m}^{\text {num }}(t)$ and $\gamma_{m}^{\text {den }}(t)$ are the posterior probability over time $t$ of the $m^{t h}$ mixture component calculated from the numerator and denominator lattices respectively, the smoothing factor $D_{m}$ is defined as $E \gamma_{m}^{\text {den }}$ and $\mathrm{E}$ is experientially set to 1 or 2 .

After we get a estimation of $\hat{W}$, we can set $\frac{\partial}{\partial \hat{H}} \mathcal{G}_{\mathrm{MWCE}}(\lambda$,

$\hat{\lambda})=0$, then the solution of $i^{t h}$ line $\hat{h}_{i}$ of the diagonal variance transform matrix $\hat{H}$ can be written as:

$$
\begin{gathered}
\hat{h}_{i}=\frac{\sum_{m}\left\{\frac{1}{\sigma_{m i}^{2}}\left[\theta_{m}^{\text {num }}\left(\hat{\mathcal{O}}_{i}^{2}\right)-\theta_{m}^{\text {den }}\left(\hat{\mathcal{O}}_{i}^{2}\right)+D_{m} K_{m i}\right]\right\}}{\sum_{m}\left(\gamma_{m}^{\text {num }}-\gamma_{m}^{\text {den }}+D_{m}\right)}, \\
K_{m i}=\tilde{\sigma}_{m i}^{2}+\left(\tilde{\mu}_{m i}-\hat{\mu}_{m i}\right)^{2} .
\end{gathered}
$$

In Eq. (9), $\tilde{\mu}_{m}$ and $\tilde{\sigma}_{m}^{2}$ are adapted by the initial transform matrices $W$ and $H$, and the other statistics are represented as:

$$
\begin{gathered}
\hat{o}(t)=o(t)-\hat{\mu}_{m}=o(t)-\hat{W} \xi_{m}, \\
\theta_{m}^{\text {num }}\left(\hat{\mathcal{O}}^{2}\right)=\sum_{r=1}^{R} \sum_{n=1}^{N_{r}} \sum_{t=t_{n}^{s}}^{t=t_{n}^{e}} \gamma_{m}^{\text {num }}(t) f^{\prime}\left(\gamma_{m}\right) \hat{o}^{2}(t), \\
\theta_{m}^{\text {den }}\left(\hat{\mathcal{O}}^{2}\right)=\sum_{r=1}^{R} \sum_{n=1}^{N_{r}} \sum_{t=t_{n}^{s}}^{t=t_{n}^{e}} \gamma_{m}^{\text {den }}(t) f^{\prime}\left(\gamma_{m}\right) \hat{o}^{2}(t) .
\end{gathered}
$$

\subsection{MWCE-MAP}

Since any function is both a weak-sense and strong-sense auxiliary function of itself around any point, it is straight forward to add a log prior distribution over the model parameters to be a weak-sense auxiliary function, and it still has a weak-sense auxiliary function for a MAP version of the original function. So a new auxiliary function could be defined as: $\mathcal{G}(\lambda, \hat{\lambda})=\mathcal{G}_{\mathrm{MWCE}}(\lambda, \hat{\lambda})+\log p(\lambda)$, where $\log p(\lambda)$ has the form as:

$$
\log p\left(\mu, \sigma^{2}\right)=\mathcal{Q}\left(\tau, \tau \mu_{\text {prior }}, \tau\left(\sigma_{\text {prior }}^{2}+\mu_{\text {prior }}^{2}\right) \mid \mu, \sigma^{2}\right),
$$

and the $\mathcal{Q}$ function is defined as:

$$
\mathcal{Q}\left(\gamma, \theta(\mathcal{O}), \theta\left(\mathcal{O}^{2}\right) \mid \mu, \sigma^{2}\right)
$$

$$
=\sum_{m}-\frac{1}{2}\left(\gamma_{m} \log \left(2 \pi \sigma^{2}\right)+\frac{\theta_{m}\left(\mathcal{O}^{2}\right)-2 \theta_{m}(\mathcal{O}) \mu_{m}+\gamma_{m} \mu_{m}^{2}}{\sigma_{m}^{2}}\right),
$$


where $\mu_{\text {prior }}$ and $\sigma_{\text {prior }}^{2}$ are estimated with conventional MAP:

$$
\begin{gathered}
\mu_{\text {prior }}=\frac{\theta_{m}^{\text {num }}(\mathcal{O})+\tau^{\mathrm{MAP}} \mu_{m}^{\text {ori }}}{\gamma_{m}^{\text {num }}+\tau^{\mathrm{MAP}}}, \\
\sigma_{\text {prior }}^{2}=\frac{\theta_{m}^{\text {num }}\left(\mathcal{O}^{2}\right)+\tau^{\mathrm{MAP}}\left(\sigma_{m}^{\text {ori2 }}+\mu_{m}^{\text {ori2 }}\right)}{\mu_{m}^{\text {num }}+\tau^{\mathrm{MAP}}}-\mu_{\text {prior }}^{2} .
\end{gathered}
$$

I-smoothing is the indispensable factor to improve the generalization when updating the parameters of the model, and it is also considered to be using a prior being based on the MLE statistics. So when applying MWCE-MAP, the numerator statistics can be updated as:

$$
\begin{gathered}
\gamma_{m}^{\text {num }}=\gamma_{m}^{\text {num }}+\tau^{I} \\
\theta_{m}^{\text {num }}(\mathcal{O})^{\prime}=\theta_{m}^{\text {num }}(\mathcal{O})+\tau^{I}\left(\mu_{\text {prior }}+\frac{\theta_{m}^{\text {num }}(\mathcal{O})}{\gamma_{m}^{\text {num }}}\right) \\
\theta_{m}^{\text {num }}\left(\mathcal{O}^{2}\right)^{\prime}=\theta_{m}^{\text {num }}\left(\mathcal{O}^{2}\right)+\tau^{I}\left(\mu_{\text {prior }}^{2}+\sigma_{\text {prior }}^{2}+\frac{\theta_{m}^{\text {num }}\left(\mathcal{O}^{2}\right)}{\gamma_{m}^{\text {num }}}\right)
\end{gathered}
$$

\section{EXPERIMENTS}

\subsection{Implementation Details}

We extend the HTK implementation to support MWCE and the other DT-based adaptation criteria, such as MMI-LR, MPE-LR, MWCELR and MWCE-MAP. Because all these criteria share most of the implementation details, we believe that this unified DT adaptation framework can provide a reasonably fair evaluation and comparison of all criteria.

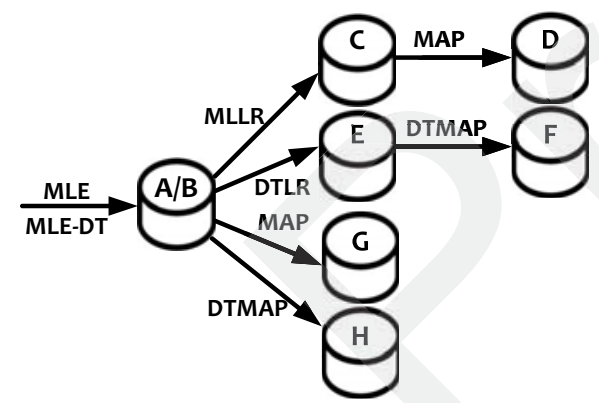

Fig.1. Experimental strategy

The baseline model of our experiments is trained on HKU96 mandarin database[11]. Totally 179 Initial-Final phones (include sil and sp) with four tones are selected to build cross-word tri-phone HMMs and the 3-state, left-to-right, no-skip topology is built for the initial phones, and 5-state for the final ones. Totally 2983 tied-states HMMs are trained, and each state has 8 Gaussian components. Fig.1. illustrates the models we constructed and compared:

Model A: the seed model using MLE training;

Model B: the seed model using DT based on model A;

Model C: the model using MLLR based on model A or B;

Model D: the model using MAP based on model C;

Model E: the model using DT-LR based on model A or B;

Model F: the model using DT-MAP based on model E;

Model G: the model using MAP based on model A or B;

Model H: the model using DT-MAP based on model A or B.

We compare the performance of MMI, MPE, MWCE criteria based LR and MAP adaptation in our experiments, so "DT-LR" in Fig.1. can be extended as "MMI-LR", and so on so forth. The lattice-based framework used in DT is also employed here, the denominator and numerator phone-level lattices are created by aligning the recognized word lattices and correcting transcriptions separately with a weakened uni-gram LM. After each iteration, the lattices should be updated with the updated models(matrices). In every adaptation processing, 3 iterations are applied and the models(matrices) with the best performance are selected to be the seed ones for next step adaptation. We just make a record of the best performance of Character Error Rate (CER\%) in each processing as shown in the tables below.

I-smoothing is applied in our experiments to improve generalization. While doing linear regression using MMI-LR,MPE-LR and MWCE-LR, the values of $\tau^{\mathrm{I}}$ are set to 50, 10 and 50, respectively. MMI-MAP and MWCE-MAP are performed with $\tau^{\mathrm{I}}=100, \tau^{\mathrm{MAP}}=25$, MPE-MAP is performed with $\tau^{\mathrm{I}}=$ $50, \tau^{\mathrm{MAP}}=25$. Finally, the weighting exponent $\alpha$ is set to $1 / 15$ in all of our experiments, for MWCE related criteria a smoothing factor was set as $\rho=0.04$.

\subsection{Adaptation on 863 mandarin database}

These experiments are tested on the evaluation set of 863 mandarin database. We randomly selected 1000 utterances form 30 persons in the training set of that database to be the adaptation data in supervised mode. A regression-class tree with 23 base classes for speech HMMs and 1 base class for non-speech ones is built.

The recognition results of the baseline model are shown in Table.1. It can be found that MWCE achieves better performance than MMI, which is comparative with MPE. The adaptation performance based on MLE seed model are shown in Table.2. It can be seen from the table that better performance can be achieved when first applying DT-LR then DT-MAP than just using DT-MAP only, and it also outperforms the process of first applying MLLR then MAP. The CER of model $F-M W C E$ is better than model $F-M P E$ for absolute $0.59 \%$ in CER, and it is also better than model $D$ for absolute $1.84 \%$ in CER. The same results are also achieved on DT based models as shown in Table.3. The model $F-M W C E$ achieves the best performance of $8.18 \%$ in CER, which outperforms any other criteria and processing modes.

\begin{tabular}{|c|c|c|c|c|}
\hline & A & B-MMI & B-MPE & B-MWCE \\
\hline CER(\%) & 21.09 & 17.58 & 14.74 & 14.58 \\
\hline
\end{tabular}

Table 1. Character Error Rate (CER\%) of the Baseline models on 863 evaluation set (e.g. B-MMI means using MMI criterion on the seed model A)

\begin{tabular}{|c|c|c|c|c|}
\hline A-base & C/D & (E/F)-MMI & (E/F)-MPE & (E/F)-MWCE \\
\hline CER $(\%)$ & $11.27 / 10.68$ & $11.02 / 9.87$ & $10.70 / 9.43$ & $10.67 / 8.84$ \\
\hline \hline A-base & G & H-MMI & H-MPE & H-MWCE \\
\hline CER $(\%)$ & 11.43 & 10.22 & 9.99 & 9.67 \\
\hline
\end{tabular}

Table 2. CER of the adaptation models on 863 evaluation set based on MLE model (e.g. (E/F)-MMI means first applying MMI-LR then MMI-MAP and MLE criterion based model A is the seed model)

\subsection{Adaptation on TsingHua mandarin database}

In these experiments we randomly select 60 persons with little accent from the TsingHua Mandarin database(Guangzhou region), for each 


\begin{tabular}{|c|c|c|c|c|}
\hline B-MMI-base & C/D & (E/F)-MMI & G & H-MMI \\
\hline CER(\%) & $10.10 / 9.86$ & $9.79 / 9.07$ & 10.11 & 9.27 \\
\hline \hline B-MPE-base & C/D & (E/F)-MPE & G & H-MPE \\
\hline CER(\%) & $9.86 / 9.72$ & $9.30 / 8.48$ & 10.28 & 8.93 \\
\hline \hline B-MWCE-base & C/D & (E/F)-MWCE & G & H-MWCE \\
\hline CER(\%) & $9.78 / 9.59$ & $9.49 / 8.18$ & 10.04 & 8.69 \\
\hline
\end{tabular}

Table 3. CER of adaptation on 863 evaluation set based on DT models

person we randomly select 15 utterances to generate the evaluation set and other 10, 20, 40 utterances are randomly selected to generate three different adaptation set respectively in supervised mode to evaluate the effect of the amount of training data. The number of regression classes are set to $16,24,32$ respectively for the different training conditions mentioned above.

As this task is a little more difficult than 863 evaluation for the affect of accent, the results are not so good as the results mentioned in section 4.2. The performance of MLE based model is $34.96 \%$ in CER, and the recognition results of adaptation based on it can be viewed in Table.4. It can be seen that with the accumulation of data for adaptation, there is little improvement achieved by MLLR and DT-LR, and the difference between them is relatively narrow. But the performance of first applying DT-LR then DT-MAP still outperforms the process of first applying MLLR then MAP. The performance of adaptation on different DT based models is shown in Table.5. The CER of the MMI-based, MPE-based and MWCEbased models are $33.19 \%, 33.02 \%$ and $32.23 \%$ respectively, where the result of MWCE is better than MPE. And the accumulating data still has limit good effect for the performance of MLLR and DT-LR. But the process of first applying MWCE-LR then MWCE-MAP still achieves the best performance of $19.98 \%$ in CER.

\begin{tabular}{|c|c|c|c|}
\hline A-base & $60 \times 10$ utts & $60 \times 20$ utts & $60 \times 40$ utts \\
\hline 34.96 & C/D & C/D & C/D \\
\hline CER $(\%)$ & $25.66 / 24.17$ & $25.05 / 23.31$ & $25.04 / 22.08$ \\
\hline \hline & (E/F)-MMI & (E/F)-MMI & (E/F)-MMI \\
\hline CER(\%) & $25.66 / 23.75$ & $25.03 / 22.31$ & $24.69 / 21.36$ \\
\hline \hline & (E/F)-MPE & (E/F)-MPE & (E/F)-MPE \\
\hline CER(\%) & $25.32 / 23.43$ & $24.82 / 22.14$ & $24.62 / 21.00$ \\
\hline \hline & (E/F)-MWCE & (E/F)-MWCE & (E/F)-MWCE \\
\hline CER(\%) & $25.58 / 23.60$ & $24.86 / 21.79$ & $24.17 / 20.92$ \\
\hline
\end{tabular}

Table 4. CER of adaptation models on TsingHua mandarin (GuangZhou region) evaluation set based on MLE model

\section{CONCLUSION}

This paper investigates on different DT adaptation criteria in supervised mode. It can be observed from the results that the estimation of the linear transformation matrices in DT criteria can achieve better performance than conventional MLLR for about 3\%-5\% in relative, but the differences between different DT-LR criteria is small. The DT-MAP criteria can achieve consistent performance improvement for both ML-based and DT-based models. For the process of first applying MWCE-LR then MWCE-MAP, a relative improvement above $10 \%$ can be achieved compared with the conventional process of first applying MLLR then MAP. It can be also found that the effect of

\begin{tabular}{|c|c|c|c|}
\hline B-MMI-base & $60 \times 10$ utts & $60 \times 20$ utts & $60 \times 40$ utts \\
\hline 33.19 & C/D & C/D & C/D \\
\hline CER(\%) & $24.57 / 23.47$ & $24.42 / 22.46$ & $24.18 / 21.64$ \\
\hline & (E/F)-MMI & (E/F)-MMI & (E/F)-MMI \\
\hline CER(\%) & $24.54 / 22.76$ & $24.35 / 21.64$ & $23.96 / 20.58$ \\
\hline \hline B-MPE-base & $60 \times 10$ utts & $60 \times 20$ utts & $60 \times 40$ utts \\
\hline 33.02 & C/D & C/D & C/D \\
\hline CER(\%) & $24.24 / 23.33$ & $24.21 / 22.09$ & $24.16 / 21.54$ \\
\hline & (E/F)-MPE & (E/F)-MPE & (E/F)-MPE \\
\hline CER(\%) & $24.22 / 22.25$ & $24.11 / 21.34$ & $23.89 / 20.29$ \\
\hline \hline B-MWCE-base & $60 \times 10$ utts & $60 \times 20$ utts & $60 \times 40$ utts \\
\hline 32.23 & C/D & C/D & C/D \\
\hline CER(\%) & $24.10 / 23.27$ & $24.16 / 22.69$ & $24.08 / 21.76$ \\
\hline & (E/F)-MWCE & $(\mathrm{E} / \mathrm{F})-\mathrm{MWCE}$ & $(\mathrm{E} / \mathrm{F})-\mathrm{MWCE}$ \\
\hline CER(\%) & $23.99 / 22.06$ & $23.72 / 21.52$ & $23.89 / 19.98$ \\
\hline
\end{tabular}

Table 5. CER of adaptation models on TsingHua mandarin (GuangZhou region) evaluation set based on DT models

accumulating adaptation data is relatively small for the DT-LR criteria, but better performance can be achieved with the accumulating of adaptation data while applying DT-MAP criteria. To evaluate these adaptation criteria on larger and more difficult tasks will be our future work

\section{REFERENCES}

[1] C.J. Leggetter, P.C. Woodland, "Maximum likelihood linear regression for speaker adaptation of continuous density HMMs," Computer Speech and Language, vol.9, pp.171-186, 1995.

[2] J.L. Gauvain, C. Lee, "Maximum A Posteriori Estimationfor Multivariate Gaussian Mixture Observations of Markov Chains," IEEE Trans.SAP, vol.2, pp.291-299, 1994.

[3] Z.J. Yan, B. Zhu, Y. Hu, R.H. Wang, "Minimum Word Classification Error Training of HMMs for Automatic Speech Recognition," in Proc. ICASSP2008, pp.4521-4524, 2008.

[4] J. Wu, Q. Huo, "A Study of Minimum Classification Error Linear Regression for Supervised Adaptation of MCE-Trained ContinuousDensity Hidden Markov Models," IEEE Trans.SAP vol.15, pp.478488, Feb, 2007.

[5] L.F. Uebel, P.C. Woodland, "Improvements in Linear Transform based Speaker Adaptation," in Proc. ICASSP2001, 2001.

[6] L. Wang, P. C. Woodland, "MPE-Based Discriminative Linear Transform for Speaker Adaptation," in Proc. ICASSP2004, pp.I321-I324, 2004.

[7] D. Povey, M.J.F. Gales, D.Y. Kim, P.C. Woodland, "MMI-MAP and MPE-MAP for Acoustic Model Adaptation," in Proc. EUROSPEECH 2003, pp.1981-1984, 2003.

[8] B.H. Juang, S. Katagiri, "Discriminative learning for minimum error classification," IEEE Transactions on Signal Processing, vol.40, no.12, pp.3043-3054, 1992.

[9] R. Schlüter, W. Macherey, B. Müller, H.Ney, "Comparison of discriminative training criteria and optimization methods for speech recognition," Speech Communication, vol.34, pp.287-310, 2001.

[10] D. Povey, "Discriminative Training for Large Vocabulary Speech Recognition," Ph.D. thesis, Cambridge University, 2004.

[11] B. Ma, Q. Huo, "Benchmark results of triphone-based acoustic modeling on HKU96 and HKU99 putonghua corpora," in Proc. ISCSLP00, pp.359-362, 2000. 